\title{
LA TERAPIA OCUPACIONAL Y LA PROMOCIÓN DE LA SALUD EMANCIPADORA PARA PERSONAS MAYORES
}

OCCUPATIONAL THERAPY AND EMANCIPATORY HEALTH PROMOTION FOR THE

ELDERLY

\section{Carmen Gloria Muñoz Muñoz ${ }^{1}$}

\begin{abstract}
RESUMEN
El ensayo revisa de manera crítica las formas de entender las estrategias de promoción de la salud que propone la Organización Mundial de la Salud para las personas mayores. Presenta diversos enfoques para de-construir el concepto y propone una forma contra hegemónica de entenderla, a partir de la subjetividad, la historicidad, la territorialidad, la capacidad de decisión y de la participación social en los procesos de salud - enfermedad - atención de las personas mayores. Desde una perspectiva teórica, se proponen algunas consideraciones para la consideración de la promoción de la salud como un campo de generación científica, que impacte en la ejecución de políticas y formación sanitaria de terapeutas ocupacionales, que gesten herramientas efectivas para el cuidado y bienestar de las personas mayores, más que sólo la replicabilidad de formas de atención en salud.
\end{abstract}

\section{PALABRAS CLAVES}

Promoción de la salud, terapia ocupacional, fortalecimiento, emancipación, personas mayores (fuente: DeCS, BIREME)

\begin{abstract}
This article provides a critical review of the understanding of health promotion strategies, as suggested by the World Health Organization for the elderly. It shows different approaches aimed at deconstructing such concept and suggests a counter-hegemonic way of understanding it, based on subjectivity, historicity, territoriality, decision-making capacity and social engagement in health-sickness-care of the elderly processes. From a theoretical standpoint, some considerations are suggested to view health promotion as a scientific generation field which can impact policy implementation and health training of occupational therapists, thereby developing effective tools for the care and wellbeing of the elderly, instead of just a replication of health care practices.
\end{abstract}

\section{KEYWORDS}

Health promotion, occupational therapy, strengthening, emancipation, the elderly (source: DeCS, BIREME)

Recibido 30/03/2017

Aceptado 17/10/2017

1 Terapeuta Ocupacional. Doctorado en Ciencias en Salud Colectiva, Universidad Autónoma Metropolitana, México. Magister en Comunicación, Universidad Astral de Chile, Licenciatura en Ciencias de la Ocupación Humana, Universidad de Chile. Académica Universidad Austral de Chile, Facultad de Medicina, Instituto de Aparato Locomotor y Rehabilitación cgmunoz@uach.cl (56) 63 2293510. ResearchedID: F-85162017. ORCID: 0000-0001-5740-6413. Dirección postal: Universidad Austral de Chile. Campus Isla Teja s/n Casa 2A, Valdivia. Región De Los Ríos, Chile. 


\section{INTRODUCCIÓN}

En nuestra época, parece no haber duda sobre la necesidad de atender el fenómeno del envejecimiento de manera integral. Actores sociales, políticos y sanitarios parecen consensuar acerca de las necesidades de este grupo etario, sobre el impacto del fenómeno de la sobrevida y los desafíos que nos presenta la curva acelerada de envejecimiento poblacional a nivel mundial (Peláez, Acosta \& Carrizo, 2015; Willing, Lanardt, \& Pereira, 2015). La intervención sanitaria nos presenta como desafío, la demanda de generar intervenciones que pongan atención a los cambio y necesidades constantes del cuerpo, la mente y el espíritu de las personas en el último tramo de la vida.

No obstante lo anterior, salvo el desarrollo para evaluación de funcionalidad y calidad de vida de las personas mayores, carecemos de lineamientos concretos para la praxis, que distingan necesidades de diversidad de colectivos en territorios concretos, determinadas por desigualdades sociales que impactan no sólo en la provisión de servicios de salud, sino de seguridad social en términos amplios. Las realidades sociales diversas imponen a buena aparte de las personas mayores de países periféricos ${ }^{2}$, desafíos de sobrevivencia, dependencia económica y precarización de la calidad de vida; a diferencia de lo que ocurre en gran parte de los países centrales, donde la problematización de la intervención en la tercera edad discurre sobre las expectativas de autocuidado, cargadas de individualismo para las decisiones correctas que allanen un buen envejecimiento o un antienvejecimiento (Jones \& Higgs, 2010). En el occidente empobrecido se tiende a invisibilizar aquello que requiere transformaciones profundas de tipo políticas, económicas y culturales.

Nuestra sociedad, atravesada por la primacía del mercado y la acumulación de bienes, parece ser incapaz de gestar una visión del bien colectivo, que atienda a la diversidad de necesidades en base al respeto del sentido social de la intervención sanitaria: la inmediatez por la disolución del síntoma, la estandarización de procedimientos, la excesiva tecnificación y tecnologización de

2 Usaré el concepto de Mario Testa, para distinguir a los "países periféricos" de los "países centrales", donde los primeros son aquellos con menor grado de dearrollo social y económico, comparativo a los "centrales", occidentales con mayores estándares de riqueza material. los procesos, atentan sobre la lógica del curso de la vida, la historización de los procesos de salud-enfermedad y el respeto por formas no tradicionales de sanación y cuidado, propias de quienes guardan un acervo valioso de formas de estar en el mundo.

En Chile, la materialización de las circunstancias antes descritas en la vida de las personas mayores, puede ser analizado a partir de dos aspectos: por una parte, está la forma occidentalizada de concebir la adultez mayor en su valor negativo, que lo vincula a carga y perjuicio, en función de lo cual no sólo se piensa, sino que se siente y se actúa ${ }^{3}$. Por otro lado (más estructural), se encuentra la política en general y la sanitaria en particular, sustentada en por la más reciente propuesta de transformación a la salud $^{4}$, que prioriza según problema de salud y su potencial costo económico. Este sistema organiza prestaciones y tiempos en rígidos protocolos con una sobrevalorización de la atención médica especializada.

Ambas hipótesis presentan un potencial explicativo cuya crítica puede conducir a nuevos tratamientos teóricos sobre el escenario social y sanitario de las necesidades de las personas mayores: la importancia de la combinación de estrategias epistemológicamente situadas en la valorización de la salud por sobre la enfermedad y en el reposicionamiento de una forma de intervención en salud que se asiente en la lógica de la territorialización y del significado de acciones cotidianas y su potencial para el bienestar. Estos principios se materializan en una forma de comprensión de la promoción de la salud (en adelante PS) desde una mirada emancipatoria y con acción colectiva, que considero enriquecería el aún incipiente tratajo de la terapia ocupacional en el área.

3 Concepto tomado de la comprensión del sociólogo francés P. Bourdieu sobre "Habitus".

4 Las Garantías Explícitas en Salud (GES), constituyen la más reciente transformación profunda al sistema de salud en Chile (inicio paulatino desde el año 2002), mediante la cual se garantiza acceso, protección financiera, oportunidad y calidad para personas que presentan algunos de los 86 problemas de salud predefinidos por la ley, indistintamente sean usuarios de un sistema de previsión público o privado. 


\section{Promoción de LA SALUd COMO PRÁCTICA SOCIAL PARA LA SALUD}

La PS tiene su origen en 1986, en la Primera Conferencia Internacional sobre la Promoción de la Salud, reunida en Otawa, Canadá (Organización Mundial de la Salud [oms], 1986). Allí, se reforzó la idea central de la Conferencia Internacional sobre Atención Primaria en Salud, realizada en el año 1978 en Alma Ata (URSs) en donde de exhortó a los países miembros a que impulsaran y pusieran en práctica la Atención Primaria de Salud (OMs, 1978). A la luz del tiempo, ambos documentos han sido pilares de la implementación de medidas sanitarias, no obstante las ideas iniciales de transformar en políticas los aprendizajes provenientes de los movimientos sociales, que ponían el poder en saberes locales para la apropiación de decisiones en salud, inspiradas, entre otros, por Freire y Werner ${ }^{5}$ (Chapela \& Cerda, 2010) se diluyeron al transformarla en una estrategia meramente educativa, dirigida a cambios de conducta en lo referido a estilos de vida perjudiciales para la salud.

La revisión de Rosales Granados y Mendoza (2016) evidencian la confusión conceptual que sitúa a la PS muchas veces como sinónimo de prevención de enfermedad, educación en salud y atención primaria en salud y la influencia de las transformaciones macroeconómicas mundiales en la planificación de acciones. Ambos elementos explican buena parte de las dificultad de implementación para muchos agentes sanitarios, que con escasos recursos intentan cumplir con estrategias económicamente infinanciables y técnicamente ambiguos, que pierden fuerza en el ejercicio pragmático de las acciones sanitarias.

La Carta de Ottawa define la PS como "el proceso de capacitar individuos y colectividades para aumentar el control sobre los determinantes de la salud y, de este modo incrementar su salud. Debiendo para esto, un individuo o grupo, ser capaz de identificar y realizar sus pretensiones, satisfacer necesidades y variar o controlar el entorno" (OMs, 1986). En el reducto de las políticas

5 Paulo Freire, educacor, brasileño, creador del método de alfabetización de adultos, centrado en las lógicas de la diálogo, la concientización y liberación, pilares de la Educación Popular. David Werner, biólogo, norteamericano, promotor y educador sobre temas de salud, rehabilitación comunitaria, concentró su trabajo en México. Escribió "Donde no hay doctor" y "Aprendiendo a Promover la Salud", entre otros. para personas mayores, se entiende como aquellas acciones que procuran el retraso de la dependencia y la discapacidad, así como la mantención de la capacidad funcional (ventilación, función muscular y rendimiento cardiovascular) (oms, 2015). Para esto, se plantea como fundamental, la atención a los factores de riesgo en la adultez: tabaquismo, el consumo de alcohol, el sedentarismo y la mala nutrición entre otros (Almeida, Castiel \& Ayres, 2009).

Esta mirada, reduce el potencial de la PS. Las condiciones sociales de vida parecen superfluas, naturalizadas y/o al margen del hecho mismo del bienestar que se alcanza fruto de un camino vital saludable, donde las condiciones de desigualdad económica y la historicidad en la construcción de la vida parece un "dato" marginal y casuístico en los lineamientos oficiales, lo que constituye incluso un caso omiso a los actuales lineamientos sobre los Determinantes Sociales de la Salud (Solar \& Irwin, 2010), donde se señala que las personas nacen, viven y mueren en circunstancias y de manera distinta según sean las condiciones sociales (estructurales e individuales) en las que se desarrollan.

En términos generales, las perspectivas hegemónicas ${ }^{6}$, ven la PS como una capacidad individual y colectiva de controlar la vida; en donde la responsabilidad de los gobiernos y otras instituciones dice relación con asegurar condiciones mínimas para el despliegue de la capacidad saludable de la población. En 1997, en la conferencia de Yakarta-Indonesia (OMs, 1997), se zanjó la necesidad de asegurar recursos para su acción, se identificó y reconoció el fuerte componente político de la estrategia, posicionando el concepto "advocacy", como una manera de referirse a algo más que "defender", sino que hablar a favor de alguien o algo, gestionar acciones y negociaciones para otros, sean estos individuos, grupos o sociedades (Chapela, 2005).

En opinión de Restrepo y Málaga (2001), esto marca un punto de inflexión entre la antigua y "nueva promoción de la salud", en donde la antigua hacia énfasis casi exclusiva a la apropiación individual de ciertos los estilos de vida, mientras que la nueva forma, da cabida al

6 Entendemos las persepctivas hegemónicas como aquellas que derivan de las políticas definidas globalmente, que buscan la estandarización y subordinación y que dominan las prácticas sanitarias locales. 
"empowerment" posibilitar/hacer visible que los individuos y los colectivos se preparen para vivir todas las etapas de la vida lidiando con las enfermedades (crónicas y lesiones) [...]" (Carvalho, 2008, p.337), mediante acciones educativas profesionales y publicitarias, en alianza de instituciones de gobierno y otras no gubernamentales.

En este contexto, resulta evidente que uno de los problemas de las prácticas de promoción con la población mayor, es que estas suelen ser organizadas de modo paternalista/maternalista, asistencialista y/o romántico/ idealista, lo que deja atrás cualquier atisbo de "apoderamiento" (Cardacci 1998, citado en Chapela, 2015). Si bien desde la teoría se entiende el reconocimiento del poder de los individuos para identificar y lograr sus aspiraciones individuales y colectivas, en lo pragmático, la participación social por ejemplo es concebida como la asistencia pasiva a un espacio de convivencia y actividad lúdico-recreativa, con escasa apropiación de poder para definir su función, lógica y proyección.

Para comprender el fenómeno de la PS desde una perspectiva distinta, como una práctica social, más que sólo una estrategia o campo de acción sanitario o una herramienta de mayor utilidad e impacto para el bienestar de personas mayores, es necesario cambiar la aproximación y de-construirla partiendo por considerar que la salud es en verdad un proceso de salud-enfermedad - atención y expresión de problemas sociales ${ }^{8}$ (Laurell, 1981). Esto permite situarla no desde la dicotomía de una condición en particular y por tanto, permite mirar siempre "la salud" aun cuando la posición es desde la relación de ésta con la enfermedad, que es, el estado frecuente de un cuerpo que acusa el proceso fisiológico normal del envejecimiento.

7 La mejor forma de traducir "empowerment" ha sido tema de discusión en la psicología y medicina comunitaria según "empoderamiento/ apoderamiento" o "fortalecimiento", diferencias implícitas en la forma de concebir las distintas formas de atribución de poder y el significado para las comunidades. Pude revisarse Montero, 2009; Silva y Martínez, 2004.

8 La comprensión de la salud desde el pensamiento de la Medicina Social y Salud Colectiva, estudia la salud-enfermedad de las colectividades como una expresión de los procesos sociales, apuntando a una integración de las ciencias sociales y biomédicas como herramientas para ello.
Esta perspectiva requiere comprender el poder de la historia del sujeto y colectivo y como esta se inscribe en la realidad humana pasada y presente, como una red de relaciones sociales donde el cuerpo es más que la presencia de un sujeto en un mundo práctico, lleno de necesidades y que aumenta progresivamente su dependencia del entorno. Es un espacio de socialización para la comprensión del presente y el futuro. Situar el deseo, la corporalidad, la concepción de realidad como herramientas para desarrollar capacidades humanas saludables en el presente y para el futuro, pero a la luz del pasado, puede ser una forma de hacer promoción de la salud más allá de la inscripción de procesos materiales y subjetivos de cuerpo individual y colectivo, que para muchos, resulta en una imposición (Chapela, 2005).

En esta misma línea, la actual operativización de la PS encuentra en la "participación social" una forma de hacer discursos y acciones. Si entendemos la participación social como un proceso complejo con diversidad de niveles y formas que van desde la búsqueda de información, generación de espacios de opinión, de disputa y de decisión sobre una arena política de intereses individuales y colectivos, más que la mera asistencia a actividades, sin duda esta tendría un mayor efecto en la sensación de bienestar y auto percepción, no sólo de la salud biológicamente entendida, sino del sentido de pertenencia y desde la valoración crítica de ese "estar".

En esta línea entonces, las diversas formas de organización de la sociedad civil, debiésemos pensarlas por tanto no sólo para la mantención de gente mayor "activa" sino como un mecanismo de información, participación y/o incidencia directa o indirecta de la política pública. Estas (las formas de organización de la sociedad civil) son vistas como una forma de legitimación de la democracia (Vázquez \& Serrano, 2005), mediadas por las posibilidades de diálogo y de discusión para un razonamiento colectivo. En Chile, abundan aquellas dedicadas a la actividades recreativas y culturales, no obstante la preocupación por temas de salud y servicios sociales en general es mínima, a pesar que estudios sobre el tema muestran que así como la morbilidad y co morbilidad es mal indicador de autopercepción, indicadores de buena autopercepción de salud lo son por ejemplo la participación social, especialmente en mujeres adultas mayores (Peláez et al., 2015). 


\section{EL VALOR DE LA PROMOCIÓN DE LA SALUD EMANCIPADORA PARA LA TERAPIA OCUPACIONAL}

La promoción de la salud puede constituir un aporte para el trabajo de Terapeutas ocupacionales, especialmente considerando el incipiente campo de la Atención Primaria para nuestra disciplina. No obstante lo anterior y a la luz de lo presentado anteriormente, es necesario comprender cada uno de los ejes de esta estrategia desde una postura crítica, que resulte en una práctica innovadora y no la mera reproducción de formas que han demostrado escasos logros y alcances para el propósito general del bienestar, entendido como aquello que sobrepasa el mero equilibrio y satisfacción de necesidades físicas, sociales, psicológicas y espirituales para el control de un fenómeno de salud particular, sino más aproximo al concepto de sumar kawsay, forma andina que puede traducirse como "estar bien", que conjuga el buen vivir con un sentido más integral, que incluye a la familia, la alimentación, el trabajo y el dominio de los conocimientos entre otros aspectos.

En esta de-construcción de la PS, es necesario considerar que el "empoderamiento" reviste dos dimensiones, una personal y una social, donde la segunda de ellas, procura una atribución de poder sobre los procesos que ocurren en el entorno de las personas y no necesaria o exclusivamente como un sentimiento de control sobre la propia vida (Carvalho, 2008). Por otra parte, comprender a las personas desde una dimensión histórica y social es reconocerles capaces de (re) organizar su presente en función de la construcción de su pasado y de acuerdo a como proyecta su futuro.

Ambas formas reconocen que el "empoderamiento" da primacía de conciencia, responsabilidad, ética, dignidad y libertad a cada sujeto/a, independiente de su condición de clase, etnia, género o edad, no sólo desde una concepción filosófica, sino como se mencionó anteriormente, llevada a la inscripción de los cuerpos. Estas concepciones dan fuerza a la idea que los seres humanos no somos sino más que una extensión de la naturaleza y por tanto podar las decisiones sobre las conductas o deseos es el efecto espejo de cuando podamos o abusamos de nuestros recursos naturales.

María Consuelo Chapela (2005), propone una forma emancipadora de entender la promoción de la salud, que parte de una manera contra - hegemónica de comprender a los seres humanos. En ésta perspectiva, se reconoce la existencia de una dimensión psico-biológica, al considerar la posibilidad de expresión del cuerpo para la satisfacción del deseo a través de la comunicación, la creatividad y otras habilidades; una dimensión política para elegir, actuar, construir alternativas y tomar decisiones; una dimensión económica y administrativa, que implica la utilización de recursos y organización de acciones; y finalmente, una dimensión técnico-práctica, que es en definitiva, el despliegue de lo que las personas hacen.

Si el sentido de las ocupaciones y la configuración de las mismas es algo dinámico a lo largo del ciclo de la vida, que depende de circunstancias vitales, de la experiencia y de las oportunidades; si la valoración del significado de las mismas es individual e intransferible, entonces, poner atención al valor de la vida pasada, de la construcción de significados sobre la salud, sobre el territorio y las experiencias asociadas a estos debiesen ser pilares de la práctica sanitaria en general y de la Terapia ocupacional en lo particular (Willing et al., 2015; Huinao, Montecinos, Pineda \& Valenzuela, 2015). Seríamos entonces responsables de entender a la ocupación como una herramienta y no sólo un constructo de valoración temporal, que tambalea entre la interpretación práctica y poética.

En esta misma línea, y ya en 1988, David Nelson señalaba que "el 'sentido' de la ocupación es enormemente retrospectivo mientras que, el 'propósito' de la ocupación es enormemente prospectivo" (Chapparo \& Ranka, 2008). Tomar como cierta esta valoración refuerza la idea de la historización, la memoria, y la remembranza como un elemento terapéutico (Salazar, 2013), que se sirve desde los macro hechos o acontecimientos contextuales a la valoración de lo cotidiano, en función de aquellos aspectos relevantes para las personas mayores en su historia.

\section{A MODO DE CONCLUSIÓN}

¿Qué puede tener de bueno vivir más de 6o años hoy en día a diferencia de hace 20 o 30 años atrás? Las circunstancias de vida no sólo condicionan la salud y la vejez, sino que la determinan. El ciclo de vida que impacta en la vejez sobrepasa la perspectiva del modelo del período crítico, o al de la acumulación de riesgos por agresiones independientes o correlacionadas, propio de formas tradicionales de entender los riesgos de la salud. 
La meta de reducir inequidades de las personas mayores no se refieren a mejorar condiciones para el tramo final de la vida, sino a la entrega de justas medidas para la atención de salud y a la evaluación de políticas y programas de salud que eliminen las desigualdades que se generan desde que respiramos en una sociedad que aún parece no comprender lo importante: que si no terminamos de generar desigualdades, jamás terminaremos de repararlas; que es necesario brindar el espacio de dialogary de opinar vinculantemente sobre nuestros cuerpos y sobre nuestros entornos.

Las y los agentes sanitarios hemos demostrado ser incapaces de "instalar hábitos saludables" en personas de edad avanzada: gran parte de nuestros usuarios se resisten para mantener sus propios ritmos de actividad (y pasividad), no comen "lo que deben", no hacen "lo que deben", entristecen y lamentamos su dependencia. Hemos sido incapaces de aprender a leer formas distintas de estar en el mundo, de andar por la vida, de lidiar con la historia; no hemos sido capaces de escuchar sus historias, de entender sus códigos, de reconocer lo que les asusta o lo que les fortalece. No hemos sido capaces de entregar de manera equitativa una forma de lidiar con sus responsabilidades, sus desventajas y sus formas (incluidas las "no saludables").

La forma hegemónica de atender las necesidades de salud de las personas mayores se ha tornado progresivamente violenta simbólica y materialmente, la estrategia de PS puede ser una eficaz herramienta terapéutica si no "sujeta a los sujetos" desde afuera o propicia la auto-sujeción, en que consumen lo que el mercado produce/oferta, pasivos y con casi nula influencia ante las imposiciones de la producción de mercancías. Fomentar el poder y la capacidad reflexiva, que se asienta en aprendizajes diversos e históricamente construidos es hacer PS, es respetar a las personas mayores y sospecho que puede ser para el resto, aprender a envejecer.

\section{ReFERENCIAS BIBLIOGRÁFICAS}

Almeida Filho, N., Castel, L. y Ayres, J. (2009). Riesgo: concepto básico de la epidemiología. En Salud colectiva, 5(3), 323-344. Recuperado de http://www.scielo.org.ar/scielo.php?script=sci_arttext\&pi$\mathrm{d}=$ S1851-82652009000300003\&lng=es\&tlng=es.

Chapela, M.C. (2005) Promoción de la salud. Un instrumento del poder y una alternativa emancipatoria. En Jarillo E. y Guinsberg E. (eds), Temas y desafíos en salud Colectiva (pp. 347-376). Buenos Aires: Lugar Editorial.

Chapela, M.C. y Cerda, A. (2010) Promoción de la salud y poder: reformulaciones desde el cuerpo-territorio y la exigibilidad de derechos. México: Universidad Autónoma Metropolitana, Unidad Xochimilco

Chaparro, C. y Ranka, J. (2008). Hacia un modelo de desempeño ocupacional: desarrollo del modelo. En Revista toc (A Curuña), 5(1), 1-44. Recuperado de: http://www.revistatog.com/num7/ pdfs/modelos1.pdf

Carvalho, S. (2008) Promoción de la Salud, "empowerment" y educación: una reflexión crítica como contribución a la reforma sanitaria. En Salud Colectiva, 4(3), 335-347. Recuperado de: http://www. scielo.org.ar/pdf/sc/v4n3/v4n3a06.pdf

Gonzalez, T., Márquez, M., Treviño, S., Gómez-Jauregui, J. y Pelcastre-Villafuerte, B. (2005). La capacidad de las Organizaciones de la Sociedad Civil para mejorar la calidad de vida de los adultos mayores: un estudio en zonas urbanas empobrecidas de México. En Salud Colectiva, 6(2), 181-194. Recuperado de: http://www. scielo.org.ar/pdf/sc/v6n2/v6n2a05.pdf

Higgs, P., Hyde, M. y Wigging, R. (2003). Researching Quality if Live Early Old Age: The importance of the Sociological Dimension. En Social Policy \& Administration, 37(3), 239-252. doi: 10.1111/14679515.00337

Huinao, M., Montecinos, C., Pineda, S. y Valenzuela, D. (2015). Construcción de la "vida ocupacional" de personas mayores en ruralidad y su influencia en la vivencia del envejecimiento. En Revista Chilena de Terapia Ocupacional, 15(1), 173-184. doi: 10.5354/0717-5346.2015.37140

Jones, I. y Higgs, P. (2010). The natural, the normal and the normative: Contested terrains in ageing and old age. En Social Science \& Medicina 77(8), 1513-1519. doi: 10.1016/j.socscimed.2010.07.022

Laurell, A (1981). La salud enfermedad como proceso social. En Revista Latinoamericana de Salud, 2(1), 7-25. Recuperado de: http://www. buenosaires.gob.ar/areas/salud/dircap/mat/matbiblio/laurell.pdf

Organización Mundial de la Salud (1978). declaración de Alma Ata. Recuperado de: http://www.paho.org/hq/index.php?option=com_docman\&task=doc_view\&gid=19004\&ltemid=2518

Organización Mundial de la Salud (1997) Declaración de Yakarta sobre la Promoción de la Salud en el Siglo xxı. Recuperado de: http:// www.who.int/healthpromotion/conferences/previous/jakarta/ en/hpr_jakarta_declaration_sp.pdf 
Organización Mundial de la Salud (1986). Carta de Ottawa para la Promoción de la Salud. Recuperado de: http://www.promocion.salud. gob.mx/dgps/descargas1/promocion/2_carta_de_ottawa.pdf

Organización Mundial de la Salud (2015). Informe mundial sobre el envejecimiento y la salud. Recuperado de: http://apps.who. int/iris/bitstream/10665/186466/1/9789240694873_spa.pdf

Peláez, E., Acosta, L. y Carrizo, E. (2015). Factores asociados a la autopercepción de salud en adultos mayores. En Revista Cubana de Salud Pública, 47(4), 638-648. Recuperado de: http://www. scielosp.org/pdf/rcsp/v41n4/spu07415.pdf

Restrepo, H. y Málaga, H. (2001) Capítulo 2: concepto y definiciones. En Promoción de la Salud: Cómo construir vida saludable. (pp. 24-33). Bogotá: Editorial Médica Panamericana.

Rosales, R., Granados, A. y Mendoza, J. (2016). Análisis crítico de las nociones de la Promoción de la Salud a partir de las Conferencias Internacionales de Promoción de la Salud. En Salud Problema / Segunda época 10(20), 30-48. Recuperado de: http://bidi.xoc. uam.mx/MostrarPDF.php

Solar, O.y Irwin, A. (2010). A Conceptual Framework for Action on the Social Determinants of Health. Recuperado de: http://www.who.int/ sdhconference/resources/ConceptualframeworkforactiononSDH_ eng.pdf

Willing, M., Lanardt, M. \& Pereira, C. (2015). Longevity according to life histories of the oldest-old. En Rev. Bras. Enfem., 68(4), 610-617. doi: http://dx.doi.org/10.1590/0034-7167.2015680418i 